\title{
The Geometry of $\mathcal{L}\left({ }^{3} l_{\infty}^{2}\right)$ and Optimal Constants in the Bohnenblust-Hille Inequality for Multilinear Forms and Polynomials
}

\author{
Sung Guen KIM* \\ Department of Mathematics, Kyungpook National University, \\ Daegu 702 -701, South Korea, sgk317@knu.ac.kr
}

Presented by Ricardo García

Received December 23, 2016

Abstract: We classify the extreme and exposed 3-linear forms of the unit ball of $\mathcal{L}\left({ }^{3} l_{\infty}^{2}\right)$. We introduce optimal constants in the Bohnenblust-Hille inequality for symmetric multilinear forms and polynomials and investigate about their relations.

Key words: Extreme points, exposed points, the optimal constants in the Bohnenblust-Hille inequality for symmetric multilinear forms and polynomials.

AMS Subject Class. (2010): 46A22.

\section{INTRODUCTION}

We write $B_{E}$ for the closed unit ball of a real Banach space $E$ and the dual space of $E$ is denoted by $E^{*} . x \in B_{E}$ is called an extreme point of $B_{E}$ if $y, z \in B_{E}$ with $x=\frac{1}{2}(y+z)$ implies $x=y=z . x \in B_{E}$ is called an exposed point of $B_{E}$ if there is a $f \in E^{*}$ so that $f(x)=1=\|f\|$ and $f(y)<1$ for every $y \in B_{E} \backslash\{x\}$. It is easy to see that every exposed point of $B_{E}$ is an extreme point. We denote by $\operatorname{ext} B_{E}$ and $\exp B_{E}$ the sets of extreme and exposed points of $B_{E}$, respectively. Let $n \in \mathbb{N}, n \geq 2$. A mapping $P: E \rightarrow \mathbb{R}$ is a continuous n-homogeneous polynomial if there exists a continuous $n$-linear form $L$ on the product $E \times \cdots \times E$ such that $P(x)=L(x, \ldots, x)$ for every $x \in E$. We denote by $\mathcal{L}\left({ }^{n} E\right)$ the Banach space of all continuous $n$-linear forms on $E$ endowed with the norm $\|L\|=\sup _{\left\|x_{j}\right\|=1,1 \leq j \leq n}\left|L\left(x_{1}, \ldots, x_{n}\right)\right| \cdot \mathcal{L}_{s}\left({ }^{n} E\right)$ denotes the closed subspace of $\mathcal{L}\left({ }^{n} E\right)$ consisting all continuous symmetric $n$-linear forms on $E$. $\mathcal{P}\left({ }^{n} E\right)$ denotes the Banach space of all continuous $n$ homogeneous polynomials from $E$ into $\mathbb{R}$ endowed with the norm $\|P\|=$

${ }^{*}$ This research was supported by the Basic Science Research Program through the National Research Foundation of Korea(NRF) funded by the Ministry of Education, Science and Technology (2013R1A1A2057788). 
$\sup _{\|x\|=1}|P(x)|$. Note that the spaces $\mathcal{L}\left({ }^{n} E\right), \mathcal{L}_{s}\left({ }^{n} E\right), \mathcal{P}\left({ }^{n} E\right)$ are very different from a geometric point of view. In particular, for integral multilinear forms and integral polynomials one has ([2], [9], [32])

$$
\operatorname{ext}_{\mathcal{L}_{I}\left({ }^{n} E\right)}=\left\{\phi_{1} \phi_{2} \cdots \phi_{n}: \phi_{i} \in \operatorname{ext} B_{E^{*}}\right\}
$$

and

$$
\operatorname{ext} B_{\mathcal{P}_{I}\left({ }^{n} E\right)}=\left\{ \pm \phi^{n}: \phi \in E^{*},\|\phi\|=1\right\},
$$

where $\mathcal{L}_{I}\left({ }^{n} E\right)$ and $\mathcal{P}_{I}\left({ }^{n} E\right)$ are the spaces of integral $n$-linear forms and integral $n$-homogeneous polynomials on $E$, respectively. For more details about the theory of multilinear mappings and polynomials on a Banach space, we refer to $[10]$.

In 1998, Choi et al. ([4], [5]) characterized the extreme points of the unit ball of $\mathcal{P}\left({ }^{2} l_{1}^{2}\right)$ and $\mathcal{P}\left({ }^{2} l_{2}^{2}\right)$. Kim [15] classified the exposed 2-homogeneous polynomials on $\mathcal{P}\left({ }^{2} l_{p}^{2}\right)(1 \leq p \leq \infty)$. Kim ([17], [19], [23]) classified the extreme, exposed, smooth points of the unit ball of $\mathcal{P}\left({ }^{2} d_{*}(1, w)^{2}\right)$, where $d_{*}(1, w)^{2}=\mathbb{R}^{2}$ with the octagonal norm of weight $w$.

In 2009, Kim [16] initiated extremal problems for bilinear forms on a classical finite dimensional real Banach space and classified the extreme, exposed, smooth points of the unit ball of $\mathcal{L}_{s}\left({ }^{2} l_{\infty}^{2}\right)$. Kim ([18], [20]-[22]) classified the extreme, exposed, smooth points of the unit balls of $\mathcal{L}_{s}\left({ }^{2} d_{*}(1, w)^{2}\right)$ and $\mathcal{L}\left({ }^{2} d_{*}(1, w)^{2}\right)$.

We refer to ([1]-[9], [11]-[32] and references therein) for some recent work about extremal properties of multilinear mappings and homogeneous polynomials on some classical Banach spaces.

Let $\mathbb{K}=\mathbb{R}$ or $\mathbb{C}$. The Bohnenblust-Hille inequality for $n$-linear forms ([3] and references therein) tells us that there exists a sequence of positive scalars $(C(n: \mathbb{K}))_{n=1}^{\infty}$ in $[1, \infty]$ such that

$$
\left(\sum_{j_{1}, \ldots, j_{n}=1}^{\infty}\left|T\left(e_{j_{1}}, \ldots, e_{j_{n}}\right)\right|^{\frac{2 n}{n+1}}\right)^{\frac{n+1}{2 n}} \leq C(n: \mathbb{K})\|T\|
$$

for all continuous $n$-linear forms $T: c_{0} \times \cdots \times c_{0} \rightarrow \mathbb{K}$. The optimal constant in the Bohnenblust-Hille inequality for $n$-linear forms $C(n: \mathbb{K})$ is defined by

$$
\begin{array}{r}
C(n: \mathbb{K}):=\sup \left\{\left(\sum_{j_{1}, \ldots, j_{n}=1}^{\infty} \mid T\left(e_{j_{1}}, \ldots, e_{j_{n}}\right)^{\frac{2 n}{n+1}}\right)^{\frac{n+1}{2 n}}:\right. \\
\left.T \in \mathcal{L}\left({ }^{n} c_{0}: \mathbb{K}\right),\|T\|=1\right\} .
\end{array}
$$


We introduce the optimal constant in the Bohnenblust-Hille inequality for symmetric $n$-linear forms $C_{s}(n: \mathbb{K})$ is defined by

$$
\begin{array}{r}
C_{s}(n: \mathbb{K}):=\sup \left\{\left(\sum_{j_{1}, \ldots, j_{n}=1}^{\infty}\left|T\left(e_{j_{1}}, \ldots, e_{j_{n}}\right)\right|^{\frac{2 n}{n+1}}\right)^{\frac{n+1}{2 n}}:\right. \\
\left.T \in \mathcal{L}_{s}\left({ }^{n} c_{0}: \mathbb{K}\right),\|T\|=1\right\} .
\end{array}
$$

It is obvious that $C_{s}(n: \mathbb{K}) \leq C(n: \mathbb{K})$. We also introduce the optimal constant in the Bohnenblust-Hille inequality for $n$-homogeneous polynomials $C_{p}(n: \mathbb{K})$ is defined by

$$
C_{p}(n: \mathbb{K}):=\sup \left\{\left(\sum_{j=1}^{\infty}\left|P\left(e_{j}\right)\right|^{\frac{2 n}{n+1}}\right)^{\frac{n+1}{2 n}}: P \in \mathcal{P}\left({ }^{n} c_{0}: \mathbb{K}\right),\|P\|=1\right\} .
$$

Recently, Diniz et al. [12] showed that $C(2: \mathbb{R})=\sqrt{2}$.

In this paper, we classify the extreme and exposed 3-linear forms of the unit ball of $\mathcal{L}\left({ }^{3} l_{\infty}^{2}\right)$. We introduce optimal constants in the Bohnenblust-Hille inequality for symmetric multilinear forms and polynomials and investigate about their relations.

\section{The eXtreme points of the Unit Ball of $\mathcal{L}\left({ }^{3} l_{\infty}^{2}\right)$}

Let $T \in \mathcal{L}\left({ }^{3} l_{\infty}^{2}\right)$ be given by

$$
\begin{aligned}
T\left(\left(x_{1}, x_{2}\right),\left(y_{1}, y_{2}\right),\left(z_{1}, z_{2}\right)\right)= & a x_{1} y_{1} z_{1}+b x_{2} y_{2} z_{2}+c_{1} x_{2} y_{1} z_{1}+c_{2} x_{1} y_{2} z_{1} \\
& +c_{3} x_{1} y_{1} z_{2}+d_{1} x_{1} y_{2} z_{2}+d_{2} x_{2} y_{1} z_{2}+d_{3} x_{2} y_{2} z_{1}
\end{aligned}
$$

for some $a, b, c_{j}, d_{j} \in \mathbb{R}$ and for $j=1,2,3$. For simplicity, we will denote $T=\left(a, b, c_{1}, c_{2}, c_{3}, d_{1}, d_{2}, d_{3}\right)$.

Theorem 2.1. Let $T=\left(a, b, c_{1}, c_{2}, c_{3}, d_{1}, d_{2}, d_{3}\right) \in \mathcal{L}\left({ }^{3} l_{\infty}^{2}\right)$. Then

$$
\begin{aligned}
\|T\|=\max \{ & \left\{\left|a+c_{1}+c_{2}+d_{3}\right|+\left|b+c_{3}+d_{1}+d_{2}\right|,\right. \\
& \left|a-c_{2}-c_{3}+d_{1}\right|+\left|b+c_{1}-d_{2}-d_{3}\right|, \\
& \left|a-b+c_{3}-d_{3}\right|+\left|c_{1}-c_{2}-d_{1}+d_{2}\right|, \\
& \left.\left|a+b-c_{1}-d_{1}\right|+\left|c_{2}-c_{3}+d_{2}-d_{3}\right|\right\} .
\end{aligned}
$$


Proof. Note that ext $B_{l_{\infty}^{2}}=\{(1,1),(1,-1),(-1,1),(-1,-1)\}$. By the KreinMilman Theorem, $B_{l_{\infty}^{2}}=\overline{c o}\left(\operatorname{ext} B_{l_{\infty}^{2}}\right)$. By the continuity and trilinearity of $T$,

$$
\begin{aligned}
& \|T\|=\max \{|T((1,1),(1,1),(1,1))|,|T((1,-1),(1,1),(1,1))| \\
& |T((1,1),(1,-1),(1,1))|,|T((1,1),(1,1),(1,-1))|, \\
& |T((1,-1),(1,-1),(1,1))|,|T((1,-1),(1,1),(1,-1))|, \\
& |T((1,1),(1,-1),(1,-1))|,|T((1,-1),(1,-1),(1,-1))|\} \\
& =\max \left\{\left|a+c_{1}+c_{2}+d_{3}\right|+\left|b+c_{3}+d_{1}+d_{2}\right|\right. \text {, } \\
& \left|a-c_{2}-c_{3}+d_{1}\right|+\left|b+c_{1}-d_{2}-d_{3}\right|, \\
& \left|a-b+c_{3}-d_{3}\right|+\left|c_{1}-c_{2}-d_{1}+d_{2}\right|, \\
& \left.\left|a+b-c_{1}-d_{1}\right|+\left|c_{2}-c_{3}+d_{2}-d_{3}\right|\right\} \text {. }
\end{aligned}
$$

Note that if $\|T\|=1$, then $|a| \leq 1,|b| \leq 1,\left|c_{j}\right| \leq 1,\left|d_{j}\right| \leq 1$, for $j=1,2,3$.

Theorem 2.2 .

$$
\begin{aligned}
e x t B_{\mathcal{L}\left({ }^{3} l_{\infty}^{2}\right)=\left\{\left(a, b, c_{1}, c_{2}, c_{3}, d_{1}, d_{2}, d_{3}\right):\right.} \\
a=\frac{1}{8}\left(\epsilon_{1}+\epsilon_{2}+\epsilon_{3}+\epsilon_{4}+\epsilon_{5}+\epsilon_{6}+\epsilon_{7}+\epsilon_{8}\right), \\
b=\frac{1}{8}\left(\epsilon_{1}-\epsilon_{2}-\epsilon_{3}-\epsilon_{4}+\epsilon_{5}+\epsilon_{6}+\epsilon_{7}-\epsilon_{8}\right), \\
c_{1}=\frac{1}{8}\left(\epsilon_{1}-\epsilon_{2}+\epsilon_{3}+\epsilon_{4}-\epsilon_{5}-\epsilon_{6}+\epsilon_{7}-\epsilon_{8}\right), \\
c_{2}=\frac{1}{8}\left(\epsilon_{1}+\epsilon_{2}-\epsilon_{3}+\epsilon_{4}-\epsilon_{5}+\epsilon_{6}-\epsilon_{7}-\epsilon_{8}\right), \\
c_{3}=\frac{1}{8}\left(\epsilon_{1}+\epsilon_{2}+\epsilon_{3}-\epsilon_{4}+\epsilon_{5}-\epsilon_{6}-\epsilon_{7}-\epsilon_{8}\right), \\
d_{1}=\frac{1}{8}\left(\epsilon_{1}+\epsilon_{2}-\epsilon_{3}-\epsilon_{4}-\epsilon_{5}-\epsilon_{6}+\epsilon_{7}+\epsilon_{8}\right), \\
d_{2}=\frac{1}{8}\left(\epsilon_{1}-\epsilon_{2}+\epsilon_{3}-\epsilon_{4}-\epsilon_{5}+\epsilon_{6}-\epsilon_{7}+\epsilon_{8}\right), \\
d_{3}=\frac{1}{8}\left(\epsilon_{1}-\epsilon_{2}-\epsilon_{3}+\epsilon_{4}+\epsilon_{5}-\epsilon_{6}-\epsilon_{7}+\epsilon_{8}\right), \\
\left.\epsilon_{j}= \pm 1, \text { for } j=1,2, \ldots, 8\right\} .
\end{aligned}
$$


Proof. Let $T=\left(a, b, c_{1}, c_{2}, c_{3}, d_{1}, d_{2}, d_{3}\right) \in \mathcal{L}\left({ }^{3} l_{\infty}^{2}\right)$ with $\|T\|=1$. Note that

$$
\begin{aligned}
T((1,1),(1,1),(1,1)) & =a+b+c_{1}+c_{2}+c_{3}+d_{1}+d_{2}+d_{3}, \\
T((1,-1),(1,1),(1,1)) & =a-b-c_{1}+c_{2}+c_{3}+d_{1}-d_{2}-d_{3}, \\
T((1,1),(1,-1),(1,1)) & =a-b+c_{1}-c_{2}+c_{3}-d_{1}+d_{2}-d_{3}, \\
T((1,1),(1,1),(1,-1)) & =a-b+c_{1}+c_{2}-c_{3}-d_{1}-d_{2}+d_{3}, \\
T((1,-1),(1,-1),(1,1)) & =a+b-c_{1}-c_{2}+c_{3}-d_{1}-d_{2}+d_{3}, \\
T((1,-1),(1,1),(1,-1)) & =a+b-c_{1}+c_{2}-c_{3}-d_{1}+d_{2}-d_{3}, \\
T((1,1),(1,-1),(1,-1)) & =a+b+c_{1}-c_{2}-c_{3}+d_{1}-d_{2}-d_{3}, \\
T((1,-1),(1,-1),(1,-1)) & =a-b-c_{1}-c_{2}-c_{3}+d_{1}+d_{2}+d_{3} .
\end{aligned}
$$

Let $A=\left(a_{i j}\right)_{1 \leq i, j \leq 8}$ be the $8 \times 8$ matrix such that

$$
\begin{aligned}
a_{i 1} & =1(i=1, \ldots, 8), & & a_{i 2}=1(i=1,5,6,7), & & a_{k 2}=-1(k=2,3,4,8), \\
a_{i 3} & =1(i=1,3,4,7), & & a_{k 3}=-1(k=2,5,6,8), & & a_{i 4}=1(i=1,2,4,6), \\
a_{k 4} & =-1(k=3,5,7,8), & a_{i 5} & =1(i=1,2,3,5), & & a_{k 5}=-1(k=4,6,7,8), \\
a_{i 6} & =1(i=1,2,7,8), & & a_{k 6}=-1(k=3,4,5,6), & & a_{i 7}=1(i=1,3,6,8), \\
a_{k 7} & =-1(k=2,4,5,7), & & a_{i 8}=1(i=1,4,5,8), & & a_{k 8}=-1(k=2,3,6,7) .
\end{aligned}
$$

By calculation, $\operatorname{det}(A)=-2^{12}$, so $A$ is invertible. Note that

$$
\begin{aligned}
A T=( & T((1,1),(1,1),(1,1)), T((1,-1),(1,1),(1,1)), \\
& T((1,1),(1,-1),(1,1)), T((1,1),(1,1),(1,-1)), \\
& T((1,-1),(1,-1),(1,1)), T((1,-1),(1,1),(1,-1)), \\
& T((1,1),(1,-1),(1,-1)), T((1,-1),(1,-1),(1,-1)))^{t}
\end{aligned}
$$

and $\|A T\|_{\infty}=\|T\|$. Note also that

$$
\begin{aligned}
T=A^{-1}( & T((1,1),(1,1),(1,1)), T((1,-1),(1,1),(1,1)), \\
& T((1,1),(1,-1),(1,1)), T((1,1),(1,1),(1,-1)), \\
& T((1,-1),(1,-1),(1,1)), T((1,-1),(1,1),(1,-1)), \\
& T((1,1),(1,-1),(1,-1)), T((1,-1),(1,-1),(1,-1)))^{t} .
\end{aligned}
$$


We claim that $T \in \operatorname{ext} B_{\mathcal{L}\left({ }^{3} l_{\infty}^{2}\right)}$ if and only if

$$
\begin{aligned}
1 & =|T((1,1),(1,1),(1,1))|=|T((1,-1),(1,1),(1,1))| \\
& =|T((1,1),(1,-1),(1,1))|=|T((1,1),(1,1),(1,-1))| \\
& =|T((1,-1),(1,-1),(1,1))|=|T((1,-1),(1,1),(1,-1))| \\
& =|T((1,1),(1,-1),(1,-1))|=|T((1,-1),(1,-1),(1,-1))| .
\end{aligned}
$$

$(\Rightarrow)$ : Otherwise. Then we have 8 cases as follows:

Case 1: $|T((1,1),(1,1),(1,1))|<1$ or

Case $2: \quad|T((1,-1),(1,1),(1,1))|<1$ or

Case 3 : $\quad|T((1,1),(1,-1),(1,1))|<1$ or

Case $4: \quad|T((1,1),(1,1),(1,-1))|<1$ or

Case 5: $\quad|T((1,-1),(1,-1),(1,1))|<1$ or

Case $6: \quad|T((1,-1),(1,1),(1,-1))|<1$ or

Case $7: \quad|T((1,1),(1,-1),(1,-1))|<1$ or

Case 8: $|T((1,-1),(1,-1),(1,-1))|<1$.

Case 1: $|T((1,1),(1,1),(1,1))|<1$. Let

$$
\begin{aligned}
& \epsilon_{1}:=T((1,1),(1,1),(1,1)), \\
& \epsilon_{2}:=T((1,-1),(1,1),(1,1)), \\
& \epsilon_{3}:=T((1,1),(1,-1),(1,1)), \\
& \epsilon_{4}:=T((1,1),(1,1),(1,-1)), \\
& \epsilon_{5}:=T((1,-1),(1,-1),(1,1)), \\
& \epsilon_{6}:=T((1,-1),(1,1),(1,-1)), \\
& \epsilon_{7}:=T((1,1),(1,-1),(1,-1)), \\
& \epsilon_{8}:=T((1,-1),(1,-1),(1,-1)) .
\end{aligned}
$$

Then,

$$
A T=\left(\epsilon_{1}, \epsilon_{2}, \epsilon_{3}, \epsilon_{4}, \epsilon_{5}, \epsilon_{6}, \epsilon_{7}, \epsilon_{8}\right)^{t} .
$$

Let $n_{0} \in \mathbb{N}$ such that $\left|\epsilon_{1}\right|+\frac{1}{n_{0}}<1$. Let $T_{1}, T_{2} \in \mathcal{L}\left({ }^{3} l_{\infty}^{2}\right)$ be the solutions of $A T_{1}=\left(\epsilon_{1}+\frac{1}{n_{0}}, \epsilon_{2}, \epsilon_{3}, \epsilon_{4}, \epsilon_{5}, \epsilon_{6}, \epsilon_{7}, \epsilon_{8}\right)^{t}, A T_{2}=\left(\epsilon_{1}-\frac{1}{n_{0}}, \epsilon_{2}, \epsilon_{3}, \epsilon_{4}, \epsilon_{5}, \epsilon_{6}, \epsilon_{7}, \epsilon_{8}\right)^{t}$. 
Note that $T_{j} \neq T,\left\|T_{j}\right\|=\left\|A T_{j}\right\|_{\infty}=1$ for $j=1,2$. It follows that

$$
A\left(\frac{1}{2}\left(T_{1}+T_{2}\right)\right)=\left(\epsilon_{1}, \epsilon_{2}, \epsilon_{3}, \epsilon_{4}, \epsilon_{5}, \epsilon_{6}, \epsilon_{7}, \epsilon_{8}\right)^{t}=A T,
$$

which shows that

$$
\frac{1}{2}\left(T_{1}+T_{2}\right)=A^{-1}\left(\epsilon_{1}, \epsilon_{2}, \epsilon_{3}, \epsilon_{4}, \epsilon_{5}, \epsilon_{6}, \epsilon_{7}, \epsilon_{8}\right)^{t}=T,
$$

so $T$ is not extreme. By the similar argument in the Case 1, if any of Cases 2-8 holds, then we may reach to a contradiction.

$(\Leftarrow)$ : Let $\epsilon_{j} \in \mathbb{R}$ be given for $j=1,2, \ldots, 8$. Consider the following system of 8 simultaneous linear equations: $A T=\left(\epsilon_{1}, \epsilon_{2}, \epsilon_{3}, \epsilon_{4}, \epsilon_{5}, \epsilon_{6}, \epsilon_{7}, \epsilon_{8}\right)^{t}$, ie,

$$
\begin{aligned}
& a+b+c_{1}+c_{2}+c_{3}+d_{1}+d_{2}+d_{3}=\epsilon_{1}, \\
& a-b-c_{1}+c_{2}+c_{3}+d_{1}-d_{2}-d_{3}=\epsilon_{2}, \\
& a-b+c_{1}-c_{2}+c_{3}-d_{1}+d_{2}-d_{3}=\epsilon_{3}, \\
& a-b+c_{1}+c_{2}-c_{3}-d_{1}-d_{2}+d_{3}=\epsilon_{4}, \\
& a+b-c_{1}-c_{2}+c_{3}-d_{1}-d_{2}+d_{3}=\epsilon_{5}, \\
& a+b-c_{1}+c_{2}-c_{3}-d_{1}+d_{2}-d_{3}=\epsilon_{6}, \\
& a+b+c_{1}-c_{2}-c_{3}+d_{1}-d_{2}-d_{3}=\epsilon_{7}, \\
& a-b-c_{1}-c_{2}-c_{3}+d_{1}+d_{2}+d_{3}=\epsilon_{8} .
\end{aligned}
$$

We get the unique solution of $(*)$ as follows: $T=A^{-1}\left(\epsilon_{1}, \epsilon_{2}, \epsilon_{3}, \epsilon_{4}, \epsilon_{5}, \epsilon_{6}, \epsilon_{7}, \epsilon_{8}\right)^{t}$, ie,

$$
\begin{aligned}
a & =\frac{1}{8}\left(\epsilon_{1}+\epsilon_{2}+\epsilon_{3}+\epsilon_{4}+\epsilon_{5}+\epsilon_{6}+\epsilon_{7}+\epsilon_{8}\right), \\
b & =\frac{1}{8}\left(\epsilon_{1}-\epsilon_{2}-\epsilon_{3}-\epsilon_{4}+\epsilon_{5}+\epsilon_{6}+\epsilon_{7}-\epsilon_{8}\right), \\
c_{1} & =\frac{1}{8}\left(\epsilon_{1}-\epsilon_{2}+\epsilon_{3}+\epsilon_{4}-\epsilon_{5}-\epsilon_{6}+\epsilon_{7}-\epsilon_{8}\right), \\
c_{2} & =\frac{1}{8}\left(\epsilon_{1}+\epsilon_{2}-\epsilon_{3}+\epsilon_{4}-\epsilon_{5}+\epsilon_{6}-\epsilon_{7}-\epsilon_{8}\right), \\
c_{3} & =\frac{1}{8}\left(\epsilon_{1}+\epsilon_{2}+\epsilon_{3}-\epsilon_{4}+\epsilon_{5}-\epsilon_{6}-\epsilon_{7}-\epsilon_{8}\right), \\
d_{1} & =\frac{1}{8}\left(\epsilon_{1}+\epsilon_{2}-\epsilon_{3}-\epsilon_{4}-\epsilon_{5}-\epsilon_{6}+\epsilon_{7}+\epsilon_{8}\right), \\
d_{2} & =\frac{1}{8}\left(\epsilon_{1}-\epsilon_{2}+\epsilon_{3}-\epsilon_{4}-\epsilon_{5}+\epsilon_{6}-\epsilon_{7}+\epsilon_{8}\right), \\
d_{3} & =\frac{1}{8}\left(\epsilon_{1}-\epsilon_{2}-\epsilon_{3}+\epsilon_{4}+\epsilon_{5}-\epsilon_{6}-\epsilon_{7}+\epsilon_{8}\right) .
\end{aligned}
$$


Let $T_{1}=\left(a+\epsilon, b+\delta, c_{1}+\gamma_{1}, c_{2}+\gamma_{2}, c_{3}+\gamma_{3}, d_{1}+\rho_{1}, d_{2}+\rho_{2}, d_{3}+\rho_{3}\right) \in \mathcal{L}\left({ }^{3} l_{\infty}^{2}\right)$ and $T_{2}=\left(a-\epsilon, b-\delta, c_{1}-\gamma_{1}, c_{2}-\gamma_{2}, c_{3}-\gamma_{3}, d_{1}-\rho_{1}, d_{2}-\rho_{2}, d_{3}-\rho_{3}\right) \in \mathcal{L}\left({ }^{3} l_{\infty}^{2}\right)$ be such that $1=\left\|T_{1}\right\|=\left\|T_{2}\right\|$ for some $\epsilon, \delta, \gamma_{j}, \rho_{j}$ for $j=1,2,3$. Then, for $k=1,2$,

$1 \geq\left|T_{k}((1,1),(1,1),(1,1))\right|=1+\left|\epsilon+\delta+\gamma_{1}+\gamma_{2}+\gamma_{3}+\rho_{1}+\rho_{2}+\rho_{3}\right|$,

$1 \geq\left|T_{k}((1,-1),(1,1),(1,1))\right|=1+\left|\epsilon-\delta-\gamma_{1}+\gamma_{2}+\gamma_{3}+\rho_{1}-\rho_{2}-\rho_{3}\right|$,

$1 \geq\left|T_{k}((1,1),(1,-1),(1,1))\right|=1+\left|\epsilon-\delta+\gamma_{1}-\gamma_{2}+\gamma_{3}-\rho_{1}+\rho_{2}-\rho_{3}\right|$,

$1 \geq\left|T_{k}((1,1),(1,1),(1,-1))\right|=1+\left|\epsilon-\delta+\gamma_{1}+\gamma_{2}-\gamma_{3}-\rho_{1}-\rho_{2}+\rho_{3}\right|$,

$1 \geq\left|T_{k}((1,-1),(1,-1),(1,1))\right|=1+\left|\epsilon+\delta-\gamma_{1}-\gamma_{2}+\gamma_{3}-\rho_{1}-\rho_{2}+\rho_{3}\right|$,

$1 \geq\left|T_{k}((1,-1),(1,1),(1,-1))\right|=1+\left|\epsilon+\delta-\gamma_{1}+\gamma_{2}-\gamma_{3}-\rho_{1}+\rho_{2}-\rho_{3}\right|$,

$1 \geq\left|T_{k}((1,1),(1,-1),(1,-1))\right|=1+\left|\epsilon+\delta+\gamma_{1}-\gamma_{2}-\gamma_{3}+\rho_{1}-\rho_{2}-\rho_{3}\right|$,

$1 \geq\left|T_{k}((1,-1),(1,-1),(1,-1))\right|=1+\left|\epsilon-\delta-\gamma_{1}-\gamma_{2}-\gamma_{3}+\rho_{1}+\rho_{2}+\rho_{3}\right|$.

Therefore, we have

$$
\begin{aligned}
& 0=\epsilon+\delta+\gamma_{1}+\gamma_{2}+\gamma_{3}+\rho_{1}+\rho_{2}+\rho_{3}, \\
& 0=\epsilon-\delta-\gamma_{1}+\gamma_{2}+\gamma_{3}+\rho_{1}-\rho_{2}-\rho_{3}, \\
& 0=\epsilon-\delta+\gamma_{1}-\gamma_{2}+\gamma_{3}-\rho_{1}+\rho_{2}-\rho_{3}, \\
& 0=\epsilon-\delta+\gamma_{1}+\gamma_{2}-\gamma_{3}-\rho_{1}-\rho_{2}+\rho_{3}, \\
& 0=\epsilon+\delta-\gamma_{1}-\gamma_{2}+\gamma_{3}-\rho_{1}-\rho_{2}+\rho_{3}, \\
& 0=\epsilon+\delta-\gamma_{1}+\gamma_{2}-\gamma_{3}-\rho_{1}+\rho_{2}-\rho_{3}, \\
& 0=\epsilon+\delta+\gamma_{1}-\gamma_{2}-\gamma_{3}+\rho_{1}-\rho_{2}-\rho_{3}, \\
& 0=\epsilon-\delta-\gamma_{1}-\gamma_{2}-\gamma_{3}+\rho_{1}+\rho_{2}+\rho_{3} .
\end{aligned}
$$

Hence, $A\left(\epsilon, \delta, \gamma_{1}, \gamma_{2}, \gamma_{3}, \rho_{1}, \rho_{2}, \rho_{3}\right)^{t}=0$. By $(* *), 0=\epsilon=\delta=\gamma_{1}=\gamma_{2}=\gamma_{3}=$ $\rho_{1}=\rho_{2}=\rho_{3}$. Hence, $T$ is extreme. Therefore, we complete the proof.

Corollary 2.3. If $T=\left(a, b, c_{1}, c_{2}, c_{3}, d_{1}, d_{2}, d_{3}\right) \in \operatorname{ext} B_{\mathcal{L}\left({ }^{3} l_{\infty}^{2}\right)}$, then $|a|$, $|b|,\left|c_{j}\right|,\left|d_{j}\right| \in\left\{0, \frac{1}{4}, \frac{1}{2}, \frac{3}{4}, 1\right\}$ for $j=1,2,3$. 
Theorem 2.4. ([26])

$$
\begin{aligned}
\operatorname{ext}_{\mathcal{L}_{s}\left({ }^{3} l_{\infty}^{2}\right)=\{} & \pm(1,0,0,0,0,0,0,0), \pm(0,1,0,0,0,0,0,0,) \\
& \pm\left(\frac{1}{2}, 0,0,0,0,-\frac{1}{2},-\frac{1}{2},-\frac{1}{2}\right), \pm\left(0, \frac{1}{2},-\frac{1}{2},-\frac{1}{2},-\frac{1}{2}, 0,0,0\right) \\
& \pm\left(\frac{1}{4},-\frac{3}{4}, \frac{1}{4}, \frac{1}{4}, \frac{1}{4}, \frac{1}{4}, \frac{1}{4}, \frac{1}{4}\right), \pm\left(-\frac{3}{4}, \frac{1}{4}, \frac{1}{4}, \frac{1}{4}, \frac{1}{4}, \frac{1}{4}, \frac{1}{4}, \frac{1}{4}\right) \\
& \pm\left(\frac{3}{4}, \frac{1}{4}, \frac{1}{4}, \frac{1}{4}, \frac{1}{4},-\frac{1}{4},-\frac{1}{4},-\frac{1}{4}\right) \\
& \left. \pm\left(\frac{1}{4}, \frac{3}{4},-\frac{1}{4},-\frac{1}{4},-\frac{1}{4}, \frac{1}{4}, \frac{1}{4}, \frac{1}{4}\right)\right\}
\end{aligned}
$$

THEOREM 2.5. $\operatorname{ext} B_{\mathcal{L}_{s}\left({ }^{3} l_{\infty}^{2}\right)}=\operatorname{ext} B_{\mathcal{L}\left({ }^{3} l_{\infty}^{2}\right)} \cap \mathcal{L}_{s}\left({ }^{3} l_{\infty}^{2}\right)$

Proof. It follows from Theorems 2.2 and 2.4.

Remarks. (1) $2^{4}=\left|\operatorname{ext} B_{\mathcal{L}_{s}\left({ }^{3} l_{\infty}^{2}\right)}\right|<\left|\operatorname{ext} B_{\mathcal{L}\left({ }^{3} l_{\infty}^{2}\right)}\right|=2^{8}$.

(2) Let $T=\left(a, b, c_{1}, c_{2}, c_{3}, d_{1}, d_{2}, d_{3}\right) \in \mathcal{L}\left({ }^{3} l_{\infty}^{2}\right)$. Then, by scaling, we may assume that $d_{j} \geq 0$ for $j=1,2,3$.

Proof. Let $T_{1}\left(\left(x_{1}, x_{2}\right),\left(y_{1}, y_{2}\right),\left(z_{1}, z_{2}\right)\right):=T\left(\left(\epsilon_{1} x_{1}, x_{2}\right),\left(\epsilon_{2} y_{1}, y_{2}\right)\right),\left(\epsilon_{3} z_{1}, z_{2}\right)$, where $\epsilon_{k}= \pm 1$ be given satisfying $\epsilon_{j} d_{j} \geq 0$ for $j=1,2,3$.

Question. Is it true that $\operatorname{ext} B_{\mathcal{L}_{s}\left({ }^{n} l_{\infty}^{2}\right)}=\operatorname{ext} B_{\mathcal{L}\left({ }^{n} l_{\infty}^{2}\right)} \cap \mathcal{L}_{s}\left({ }^{n} l_{\infty}^{2}\right)$ for $n \geq 4$ ?

3. The EXposed points of the Unit BALL of $\mathcal{L}\left({ }^{3} l_{\infty}^{2}\right)$

Theorem 3.1. Let $f \in \mathcal{L}\left({ }^{3} l_{\infty}^{2}\right)^{*}$ with

$$
\begin{aligned}
& \alpha=f\left(x_{1} y_{1} z_{1}\right), \quad \beta=f\left(x_{2} y_{2} z_{2}\right), \quad \gamma_{1}=f\left(x_{2} y_{1} z_{1}\right), \quad \gamma_{2}=f\left(x_{1} y_{2} z_{1}\right), \\
& \gamma_{3}=f\left(x_{1} y_{1} z_{2}\right), \quad \delta_{1}=f\left(x_{1} y_{2} z_{2}\right), \quad \delta_{2}=f\left(x_{2} y_{1} z_{2}\right), \quad \delta_{3}=f\left(x_{2} y_{2} z_{1}\right) .
\end{aligned}
$$


Then,

$$
\begin{aligned}
\|f\|=\max \left\{\left|a \alpha+b \beta+\sum_{j=1,2,3} c_{j} \gamma_{j}+\sum_{j=1,2,3} d_{j} \delta_{j}\right|:\right. \\
a=\frac{1}{8}\left(\epsilon_{1}+\epsilon_{2}+\epsilon_{3}+\epsilon_{4}+\epsilon_{5}+\epsilon_{6}+\epsilon_{7}+\epsilon_{8}\right), \\
b=\frac{1}{8}\left(\epsilon_{1}-\epsilon_{2}-\epsilon_{3}-\epsilon_{4}+\epsilon_{5}+\epsilon_{6}+\epsilon_{7}-\epsilon_{8}\right), \\
c_{1}=\frac{1}{8}\left(\epsilon_{1}-\epsilon_{2}+\epsilon_{3}+\epsilon_{4}-\epsilon_{5}-\epsilon_{6}+\epsilon_{7}-\epsilon_{8}\right), \\
c_{2}=\frac{1}{8}\left(\epsilon_{1}+\epsilon_{2}-\epsilon_{3}+\epsilon_{4}-\epsilon_{5}+\epsilon_{6}-\epsilon_{7}-\epsilon_{8}\right), \\
c_{3}=\frac{1}{8}\left(\epsilon_{1}+\epsilon_{2}+\epsilon_{3}-\epsilon_{4}+\epsilon_{5}-\epsilon_{6}-\epsilon_{7}-\epsilon_{8}\right), \\
d_{1}=\frac{1}{8}\left(\epsilon_{1}+\epsilon_{2}-\epsilon_{3}-\epsilon_{4}-\epsilon_{5}-\epsilon_{6}+\epsilon_{7}+\epsilon_{8}\right), \\
d_{2}=\frac{1}{8}\left(\epsilon_{1}-\epsilon_{2}+\epsilon_{3}-\epsilon_{4}-\epsilon_{5}+\epsilon_{6}-\epsilon_{7}+\epsilon_{8}\right), \\
d_{3}=\frac{1}{8}\left(\epsilon_{1}-\epsilon_{2}-\epsilon_{3}+\epsilon_{4}+\epsilon_{5}-\epsilon_{6}-\epsilon_{7}+\epsilon_{8}\right), \\
\left.\epsilon_{j}= \pm 1, \text { for } j=1,2, \ldots, 8\right\}
\end{aligned}
$$

Proof. Proof. It follows from Theorem 2.2 and the Krein-Milman Theorem.

THEOREM 3.2. $\exp B_{\mathcal{L}\left({ }^{3} l_{\infty}^{2}\right)}=\operatorname{ext} B_{\mathcal{L}\left({ }^{3} l_{\infty}^{2}\right)}$.

Proof. We will show that $\operatorname{ext} B_{\mathcal{L}\left({ }^{3} l_{\infty}^{2}\right)} \subset \exp B_{\left.\mathcal{L}^{3}{ }^{3} l_{\infty}^{2}\right)}$. By Theorem 2.2, Corollary 2.3 and $\operatorname{Remarks}(2)$, it suffices to show that if

$$
\begin{aligned}
T= & (1,0,0,0,0,0,0,0),\left(-\frac{1}{2}, \frac{1}{2}, \frac{1}{2}, \frac{1}{2}, 0,0,0,0\right) \\
& \left(-\frac{3}{4}, \frac{1}{4}, \frac{1}{4}, \frac{1}{4}, \frac{1}{4}, \frac{1}{4}, \frac{1}{4}, \frac{1}{4}\right),\left(\frac{3}{4},-\frac{1}{4},-\frac{1}{4},-\frac{1}{4}, \frac{1}{4}, \frac{1}{4}, \frac{1}{4}, \frac{1}{4}\right),
\end{aligned}
$$

then $T \in \exp B_{\mathcal{L}\left({ }^{3} l_{\infty}^{2}\right)}$.

Claim: $T=(1,0,0,0,0,0,0,0) \in \exp B_{\mathcal{L}\left({ }^{3} l_{\infty}^{2}\right)}$.

Let $f \in \mathcal{L}\left({ }^{3} l_{\infty}^{2}\right)^{*}$ with $\alpha=1,0=\beta=\gamma_{j}=\delta_{j}$ for $j=1,2,3$. Note that, by Corollary 2.3 and Theorems 2.2 and 3.1, $\|f\|=1=f(T)$ and $|f(S)|<1$ for all $S \in \operatorname{ext} B_{\mathcal{L}\left({ }^{3} l_{\infty}^{2}\right)} \backslash\{ \pm T\}$. The claim follows from Theorem 2.3 of [21]. 
Claim: $T=\left(-\frac{1}{2}, \frac{1}{2}, \frac{1}{2}, \frac{1}{2}, 0,0,0,0\right) \in \exp B_{\mathcal{L}\left({ }^{3} l_{\infty}^{2}\right)}$.

Let $f \in \mathcal{L}\left({ }^{3} l_{\infty}^{2}\right) *$ with $-\alpha=\frac{1}{2}=\beta=\gamma_{1}=\gamma_{2}, 0=\gamma_{3}=\delta_{j}$ for $j=1,2,3$. Note that, by Corollary 2.3 and Theorems 2.2 and 3.1, $\|f\|=1=f(T)$ and $|f(S)|<1$ for all $S \in \operatorname{ext} B_{\mathcal{L}\left({ }^{3} l_{\infty}^{2}\right)} \backslash\{ \pm T\}$. The claim follows from Theorem 2.3 of $[21]$.

Claim: $T=\left(-\frac{3}{4}, \frac{1}{4}, \frac{1}{4}, \frac{1}{4}, \frac{1}{4}, \frac{1}{4}, \frac{1}{4}, \frac{1}{4}\right) \in \exp B_{\mathcal{L}\left({ }^{3} l_{\infty}^{2}\right)}$.

Let $f \in \mathcal{L}\left({ }^{3} l_{\infty}^{2}\right)^{*}$ with $\alpha=-\frac{1}{2}, \frac{5}{14}=\beta=\gamma_{j}=\delta_{j}$ for $j=1,2,3$. Note that, by Corollary 2.3 and Theorems 2.2 and 3.1, $\|f\|=1=f(T)$ and $|f(S)|<1$

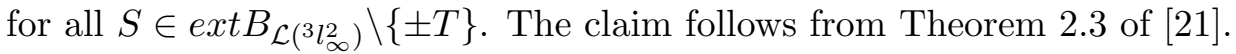

Claim: $T=\left(\frac{3}{4},-\frac{1}{4},-\frac{1}{4},-\frac{1}{4}, \frac{1}{4}, \frac{1}{4}, \frac{1}{4}, \frac{1}{4}\right) \in \exp B_{\mathcal{L}\left({ }^{3} l_{\infty}^{2}\right)}$.

Let $f \in \mathcal{L}\left({ }^{3} l_{\infty}^{2}\right)^{*}$ with $\alpha=\frac{1}{2},-\frac{5}{14}=\beta=\gamma_{1}=\gamma_{2}=-\gamma_{3}=-\delta_{j}$ for $j=$ $1,2,3$. Note that, by Corollary 2.3 and Theorems 2.2 and $3.1,\|f\|=1=f(T)$

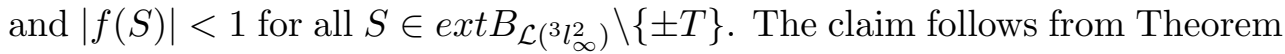
2.3 of $[21]$. We complete the proof.

TheOREM 3.3. $([26]) \exp B_{\mathcal{L}_{s}\left({ }^{3} l_{\infty}^{2}\right)}=\operatorname{ext} B_{\mathcal{L}_{s}\left({ }^{3} l_{\infty}^{2}\right)}$.

TheOREM 3.4. $\exp B_{\mathcal{L}_{s}\left({ }^{3} l_{\infty}^{2}\right)}=\exp B_{\mathcal{L}\left({ }^{3} l_{\infty}^{2}\right)} \cap \mathcal{L}_{s}\left({ }^{3} l_{\infty}^{2}\right)$.

Proof. It follows from Theorems 3.2 and 3.3.

Question. Is it true that $\exp B_{\mathcal{L}_{s}\left({ }^{n} l_{\infty}^{2}\right)}=\exp B_{\mathcal{L}\left({ }^{n} l_{\infty}^{2}\right)} \cap \mathcal{L}_{s}\left({ }^{n} l_{\infty}^{2}\right)$ for $n \geq 4$ ?

4. Optimal constants in the Bohnenblust-Hille inequality for SYMMETRIC MULTILINEAR FORMS AND POLYNOMIALS

Theorem 4.1. $1 \leq C_{p}(n: \mathbb{K}) \leq \frac{n^{n}}{n !} C_{s}(n: \mathbb{K}) \leq \frac{n^{n}}{n !} C(n: \mathbb{K})$ for all $n \geq 2$.

Proof. It is enough to show that $C_{p}(n: \mathbb{K}) \leq \frac{n^{n}}{n !} C_{s}(n: \mathbb{K})$. Let $P \in \mathcal{P}\left({ }^{n} c_{0}\right.$ : $\mathbb{K}),\|P\|=1$. By the polarization formula, $\|\check{P}\| \leq \frac{n^{n}}{n !}\|P\|=\frac{n^{n}}{n !}$, where $\check{P}$ is the corresponding symmetric $n$-linear form to $P$. Hence,

$$
\begin{aligned}
\left(\sum_{j=1}^{\infty}\left|\frac{n !}{n^{n}} P\left(e_{j}\right)\right|^{\frac{2 n}{n+1}}\right)^{\frac{n+1}{2 n}} & \leq\left(\sum_{j_{1}, \cdots, j_{n}=1}^{\infty}\left|\frac{n !}{n^{n}} \check{P}\left(e_{j_{1}}, \cdots, e_{j_{n}}\right)\right|^{\frac{2 n}{n+1}}\right)^{\frac{n+1}{2 n}} \\
& \leq C_{s}(n: \mathbb{K}) .
\end{aligned}
$$


TheOREM 4.2. $C_{s}(2: \mathbb{R})=C(2: \mathbb{R})=\sqrt{2}$.

Proof. It is enough to show that $C_{s}(2: \mathbb{R})=\sqrt{2}$. Let

$$
T\left(\left(x_{1}, x_{2}\right),\left(y_{1}, y_{2}\right)\right)=\frac{1}{2} x_{1} y_{1}-\frac{1}{2} x_{2} y_{2}+\frac{1}{2} x_{1} y_{2}+\frac{1}{2} x_{2} y_{1} .
$$

Then $T \in \mathcal{L}_{s}\left({ }^{2} l_{\infty}^{2}\right),\|T\|=1$. By a result of $[12], \sqrt{2} \leq\left(\sum_{i, j=1}^{2}\left|T\left(e_{i}, e_{j}\right)\right|^{\frac{4}{3}}\right)^{\frac{3}{4}} \leq$ $C_{s}(2: \mathbb{R}) \leq C(2: \mathbb{R})=\sqrt{2}$.

THEOREM 4.3. ([16])

$$
\operatorname{ext} B_{\mathcal{L}_{s}\left({ }^{2} l_{\infty}^{2}\right)}=\left\{ \pm(1,0,0,0), \pm(0,1,0,0), \pm \frac{1}{2}(1,-1,1,1), \pm \frac{1}{2}(1,-1,-1,-1)\right\} .
$$

Theorem 4.4 .

$$
\begin{aligned}
\sup & \left\{\left(\sum_{i, j=1}^{2}\left|T\left(e_{i}, e_{j}\right)\right|^{\frac{4}{3}}\right)^{\frac{3}{4}}: T \in \mathcal{L}_{s}\left({ }^{2} l_{\infty}^{2}\right),\|T\|=1, T \notin \operatorname{ext} B_{\mathcal{L}_{s}\left(2 l_{\infty}^{2}\right)}\right\} \\
& =C_{s}(2: \mathbb{R}) .
\end{aligned}
$$

Proof. Let

$$
l:=\sup \left\{\left(\sum_{i, j=1}^{2}\left|T\left(e_{i}, e_{j}\right)\right|^{\frac{4}{3}}\right)^{\frac{3}{4}}: T \in \mathcal{L}_{s}\left({ }^{2} l_{\infty}^{2}\right),\|T\|=1, T \notin \operatorname{ext} B_{\mathcal{L}_{s}\left({ }^{2} l_{\infty}^{2}\right)}\right\} .
$$

For $|c|<\frac{1}{2}$, let

$$
T_{c}\left(\left(x_{1}, x_{2}\right),\left(y_{1}, y_{2}\right)\right)=\frac{1}{2} x_{1} y_{1}-\frac{1}{2} x_{2} y_{2}+c x_{1} y_{2}+c x_{2} y_{1} .
$$

Then $T_{c} \in \mathcal{L}_{s}\left({ }^{2} l_{\infty}^{2}\right),\left\|T_{c}\right\|=1$. By Theorem $4.3, T_{c} \notin \operatorname{ext} B_{\mathcal{L}_{s}\left({ }^{2} l_{\infty}^{2}\right)}$. It follows that

$$
\begin{aligned}
C_{s}(2: \mathbb{R}) & \geq l \geq \sup \left\{\left(\sum_{i, j=1}^{2}\left|T_{c}\left(e_{i}, e_{j}\right)\right|^{\frac{4}{3}}\right)^{\frac{3}{4}}:|c|<\frac{1}{2}\right\} \\
& =\sup _{|c|<\frac{1}{2}}\left(2\left(\frac{1}{2}\right)^{\frac{4}{3}}+2|c|^{\frac{4}{3}}\right)^{\frac{3}{4}}=\sqrt{2}=C_{s}(2: \mathbb{R}) .
\end{aligned}
$$


Theorem 4.5. Let $n \geq 2$. Then, $2^{\frac{n+1}{2 n}} \leq C_{p}(n: \mathbb{R})$.

Proof. Let

$$
\begin{aligned}
w:=\sup \left\{\left(\sum_{j=1}^{\infty}\left|P\left(e_{j}\right)\right|^{\frac{2 n}{n+1}}\right)^{\frac{n+1}{2 n}}: P \in \mathcal{P}\left({ }^{n} c_{0}\right),\|P\|=1,\right. & \\
& \left.P\left(\left(x_{m}\right)_{m=1}^{\infty}\right)=\sum_{j=1}^{\infty} a_{j} x_{j}^{n} \text { for some } a_{j} \in \mathbb{R}\right\} .
\end{aligned}
$$

Claim: $w=2^{\frac{n+1}{2 n}}$.

Let $P \in \mathcal{P}\left({ }^{n} c_{0}\right),\|P\|=1, P\left(\left(x_{m}\right)_{m=1}^{\infty}\right)=\sum_{j=1}^{\infty} a_{j} x_{j}^{n}$ for some $a_{j} \in \mathbb{R}$. Let $A:=\left\{j \in \mathbb{N}: a_{j} \geq 0\right\}$ and $B:=\mathbb{N} \backslash A$. Note that, for every $k \in \mathbb{N}$,

$$
1 \geq\left|P\left(\sum_{j \in A, j \leq k} e_{j}\right)\right|=\sum_{j \in A, j \leq k}\left|a_{j}\right|
$$

and

$$
1 \geq\left|P\left(\sum_{j \in B, j \leq k} e_{j}\right)\right|=\sum_{j \in B, j \leq k}\left|a_{j}\right| .
$$

Hence,

$$
\sum_{j \in A}\left|a_{j}\right| \leq 1, \quad \sum_{j \in B}\left|a_{j}\right| \leq 1
$$

It follows that

$$
\begin{aligned}
\left(\sum_{j=1}^{\infty}\left|P\left(e_{j}\right)\right|^{\frac{2 n}{n+1}}\right)^{\frac{n+1}{2 n}} & =\left(\sum_{j \in A}\left|a_{j}\right|^{\frac{2 n}{n+1}}+\sum_{j \in B}\left|a_{j}\right|^{\frac{2 n}{n+1}}\right)^{\frac{n+1}{2 n}} \\
& \leq\left(\sum_{j \in A}\left|a_{j}\right|+\sum_{j \in B}\left|a_{j}\right|\right)^{\frac{n+1}{2 n}} \\
& \leq 2^{\frac{n+1}{2 n}}
\end{aligned}
$$

which shows that $w \leq 2^{\frac{n+1}{2 n}}$. Let $P_{0}\left(\left(x_{m}\right)_{m=1}^{\infty}\right)=x_{1}^{n}-x_{2}^{n} \in \mathcal{P}\left({ }^{n} c_{0}\right)$ for $\left(x_{m}\right)_{m=1}^{\infty} \in c_{0}$. Then $\left\|P_{0}\right\|=1$. Hence,

$$
2^{\frac{n+1}{2 n}}=\left(\sum_{j=1}^{\infty}\left|P_{0}\left(e_{j}\right)\right|^{\frac{2 n}{n+1}}\right)^{\frac{n+1}{2 n}} \leq w \leq 2^{\frac{n+1}{2 n}} .
$$

Therefore, $2^{\frac{n+1}{2 n}} \leq C_{p}(n: \mathbb{R})$. We complete the proof. 
Corollary 4.6. $C(2: \mathbb{R})<2^{\frac{3}{4}} \leq C_{p}(2: \mathbb{R}) \leq 2 \sqrt{2}$.

THEOREM 4.7. Let $T: l_{\infty}^{2}(\mathbb{R}) \times l_{\infty}^{2}(\mathbb{R}) \rightarrow \mathbb{R}$ be given by $T(x, y)=$ $\sum_{i, j=1}^{2} a_{i j} x_{i} y_{j}$, with $a_{i j} \in \mathbb{R}, a_{12}=a_{21}$. Then the symmetric bilinear forms satisfying

$$
\left(\sum_{i, j=1}^{2}\left|T\left(e_{i}, e_{j}\right)\right|^{\frac{4}{3}}\right)^{\frac{3}{4}}=\sqrt{2}\|T\|
$$

are given by $T(x, y)=a\left(x_{1} y_{1}-x_{2} y_{2}+x_{1} y_{2}+x_{2} y_{1}\right)$ or $T(x, y)=a\left(-x_{1} y_{1}+\right.$ $\left.x_{2} y_{2}+x_{1} y_{2}+x_{2} y_{1}\right)$ for all $a \in \mathbb{R} \backslash\{0\}$.

Proof. It follows from Theorem 4.1 of [3]. Then,

Theorem 4.8. Let $T \in \mathcal{L}_{s}\left({ }^{2} l_{\infty}^{2}\right),\|T\|=1, T\left(e_{j}, e_{j}\right) \neq 0$ for $j=1,2$.

$$
\left(\sum_{i, j=1}^{2}\left|T\left(e_{i}, e_{j}\right)\right|^{\frac{4}{3}}\right)^{\frac{3}{4}}=C_{s}(2: \mathbb{R})
$$

if and only if $T \in \operatorname{ext} B_{\mathcal{L}_{s}\left({ }^{2} l_{\infty}^{2}\right)}$.

Proof. It follows from Theorems 4.2, 4.3 and 4.7.

THEOREM 4.9 .

$$
\sup \left\{\left(\sum_{i, j=1}^{2}\left|T\left(e_{i}, e_{j}\right)\right|^{\frac{6}{4}}\right)^{\frac{4}{6}}: T \in \operatorname{ext} B_{\mathcal{L}\left(3 l_{\infty}^{2}\right)}\right\}=\frac{\left(7+3^{\frac{3}{2}}\right)^{\frac{2}{3}}}{4}<C(3: \mathbb{R}) .
$$

Proof. Diniz et al. [12] showed that $2^{\frac{2}{3}} \leq C(3: \mathbb{R}) \leq 1.782$. By Theorem 2.2 and Corollary 2.3,

$$
\sup \left\{\left(\sum_{i, j=1}^{2}\left|T\left(e_{i}, e_{j}\right)\right|^{\frac{6}{4}}\right)^{\frac{4}{6}}: T \in \operatorname{ext} B_{\mathcal{L}\left({ }^{3} l_{\infty}^{2}\right)}\right\}=\frac{\left(7+3^{\frac{3}{2}}\right)^{\frac{2}{3}}}{4}<2^{\frac{2}{3}} \leq C(3: \mathbb{R})
$$

Questions. (1) Is it true that $C_{s}(n: \mathbb{R})=C(n: \mathbb{R})$ for all $n \geq 3$ ?

(2) Is it true that $C_{p}(2: \mathbb{R})=2^{\frac{3}{4}}$ ? 


\section{REFERENCES}

[1] R.M. Aron, Y.S. Choi, S.G. Kim, M. Maestre, Local properties of polynomials on a Banach space, Illinois J. Math. 45 (1) (2001), 25-39.

[2] C. Boyd, R.A. RyAn, Geometric theory of spaces of integral polynomials and symmetric tensor products, J. Funct. Anal. 179 (1) (2001), 18-42.

[3] W. Cavalcante, D. Pellegrino, Geometry of the closed unit ball of the space of bilinear forms on $l_{\infty}^{2}$, arXiv:1603.01535v2.

[4] Y.S. ChOI, H. KI, S.G. KIM, Extreme polynomials and multilinear forms on $l_{1}$, J. Math. Anal. Appl. 228 (2) (1998), 467-482.

[5] Y.S. Choi, S.G. KIM, The unit ball of $\mathcal{P}\left({ }^{2} l_{2}^{2}\right)$, Arch. Math. (Basel) $\mathbf{7 1}(6)$ (1998), $472-480$.

[6] Y.S. Choi, S.G. KIm, Extreme polynomials on $c_{0}$, Indian J. Pure Appl. Math. 29 (10) (1998), 983-989.

[7] Y.S. ChOI, S.G. KIM, Smooth points of the unit ball of the space $\mathcal{P}\left({ }^{2} l_{1}\right)$, Results Math. 36 (1-2) (1999), 26-33.

[8] Y.S. CHoI, S.G. KIM, Exposed points of the unit balls of the spaces $\mathcal{P}\left({ }^{2} l_{p}^{2}\right)(p=1,2, \infty)$, Indian J. Pure Appl. Math. 35 (1) (2004), 37-41.

[9] V. Dimant, D. Galicer, R. García, Geometry of integral polynomials, $M$-ideals and unique norm preserving extensions, J. Funct. Anal. $262(5)$ (2012), $1987-2012$.

[10] S. Dineen, "Complex Analysis on Infinite Dimensional Spaces", SpringerVerlag, London, 1999.

[11] S. DineEn, Extreme integral polynomials on a complex Banach space, Math. Scand. 92 (1) (2003), 129-140.

[12] D. Diniz, G.A. Muñoz-Fernández, D. Pellegrino, J.B. SeonneSEPÚLVEDA, Lower bounds for the constants in the Bohnenblust-Hille inequality: the case of real scalars, Proc. Amer. Math. Soc. 142 (2) (2014), $575-580$.

[13] B.C. GrecU, Geometry of 2-homogeneous polynomials on $l_{p}$ spaces, $1<p<$ क, J. Math. Anal. Appl. 273 (2) (2002), 262-282.

[14] B.C. Grecu, G.A. Muñoz-Fernández, J.B. Seonne-Sepúlveda, Unconditional constants and polynomial inequalities, J. Approx. Theory $161(2)$ (2009), $706-722$.

[15] S.G. KIM, Exposed 2-homogeneous polynomials on $\mathcal{P}\left({ }^{2} l_{p}^{2}\right)(1 \leq p \leq \infty)$, Math. Proc. R. Ir. Acad. 107A (2) (2007), 123-129.

[16] S.G. KIM, The unit ball of $\mathcal{L}_{s}\left({ }^{2} l_{\infty}^{2}\right)$, Extracta Math. 24(1) (2009), 17-29.

[17] S.G. KIm, The unit ball of $\mathcal{P}\left({ }^{2} d_{*}(1, w)^{2}\right)$, Math. Proc. R. Ir. Acad. $111 \mathrm{~A}(2)$ (2011), $79-94$.

[18] S.G. KIM, The unit ball of $\mathcal{L}_{s}\left({ }^{2} d_{*}(1, w)^{2}\right)$, Kyungpook Math. J. 53 (2) (2013), $295-306$

[19] S.G. KIM, Smooth polynomials of $\mathcal{P}\left({ }^{2} d_{*}(1, w)^{2}\right)$, Math. Proc. R. Ir. Acad. 113A (1) (2013), 45-58.

[20] S.G. KIM, Extreme bilinear forms of $\mathcal{L}\left({ }^{2} d_{*}(1, w)^{2}\right)$, Kyungpook Math. J. $53(4)$ (2013), 625-638. 
[21] S.G. KIm, Exposed symmetric bilinear forms of $\mathcal{L}_{s}\left({ }^{2} d_{*}(1, w)^{2}\right)$, Kyungpook Math. J. $54(3)$ (2014), 341-347.

[22] S.G. KIM, Exposed bilinear forms of $\mathcal{L}\left({ }^{2} d_{*}(1, w)^{2}\right)$, Kyungpook Math. J. 55 (1) (2015), $119-126$

[23] S.G. KIM, Exposed 2-homogeneous polynomials on the 2-dimensional real predual of Lorentz sequence space, Mediterr. J. Math. 13 (5) (2016), 2827-2839.

[24] S.G. KIM, The geometry of $\mathcal{L}\left({ }^{2} l_{\infty}^{2}\right)$, to appear in Kyungpook Math. J. 58 (2018).

[25] S.G. KIM, The unit ball of $\mathcal{L}_{s}\left({ }^{2} l_{\infty}^{3}\right)$, Comment. Math. Prace Mat. 57 (1) (2017), $1-7$.

[26] S.G. KIM, The geometry of $\mathcal{L}_{s}\left({ }^{3} l_{\infty}^{2}\right)$, Commun. Korean Math. Soc. $32(4)$ (2017), 991-997.

[27] S.G. KIM, S.H. LEE, Exposed 2-homogeneous polynomials on Hilbert spaces, Proc. Amer. Math. Soc. 131 (2) (2003), 449-453.

[28] J. Lee, K.S. Rim, Properties of symmetric matrices, J. Math. Anal. Appl. 305 (1) (2005), $219-226$.

[29] G.A. Muñoz-Fernández, S. Révész, J.B. Seoane-Sepúlveda, Geometry of homogeneous polynomials on non symmetric convex bodies, Math. Scand. 105 (1) (2009), 147-160.

[30] G.A. Muñoz-Fernández, J.B. Seonne-Sepúlveda, Geometry of Banach spaces of trinomials, J. Math. Anal. Appl. 340 (2) (2008), 1069-1087.

[31] R.A. Ryan, B. Turett, Geometry of spaces of polynomials, J. Math. Anal. Appl. 221 (2) (1998), 698-711.

[32] W.M. Ruess, C.P. Stegall, Extreme points in duals of operator spaces, Math. Ann. 261 (4) (1982), 535-546. 